in vivo $34: 1063-1069$ (2020)

doi:10.21873/invivo.11876

\title{
Student Views on a Novel Holistic Surgical Education Curriculum (iG4): A Multi-national Survey in a Changing Landscape
}

\author{
MICHAIL SIDERIS ${ }^{1 *}$, MARIOS NICOLAIDES ${ }^{2 *}$, IAKOVOS THEODOULOU $^{3 *}$, ELIF ILIRIA EMIN $^{3}$, \\ JOHN GERRARD HANRAHAN ${ }^{4}$, AIKATERINI DEDEILIA ${ }^{5}$, EFTHYMIA THEODOROU $^{6}$, \\ GEORGIOS PAPAROIDAMIS $^{7}$, ZIENA ABDULLAH ${ }^{8}$, CONSTANTINOS PAPOUTSOS $^{6}$, \\ THEODOROS PITTARAS $^{9}$, FUNLAYO ODEJINMI ${ }^{10}$ and APOSTOLOS PAPALOIS ${ }^{11,12}$ \\ ${ }^{1}$ Women's Health Research Unit, Queen Mary University of London, London, U.K.; \\ ${ }^{2}$ Barts Cancer Institute, Queen Mary University of London, London, U.K.; \\ ${ }^{3}$ Faculty of Life Sciences and Medicine, King's College London, London, U.K.; \\ ${ }^{4}$ East of Anglia Deanery, Cambridge, U.K.; \\ ${ }^{5}$ Medical School, National and Kapodistrian University of Athens, Athens, Greece; \\ ${ }^{6}$ Barts and The London School of Medicine and Dentistry, Queen Mary University of London, London, U.K.; \\ ${ }^{7}$ Medical School, Aristotle University of Thessaloniki, Thessaloniki, Greece; \\ ${ }^{8}$ Barking, Havering and Redbridge Hospitals NHS Trust, London, U.K.; \\ ${ }^{9}$ Hematology Laboratory-Blood Bank, Aretaieion Hospital, University of Athens School of Medicine, Athens, Greece; \\ ${ }^{10}$ Whipps Cross University Hospital, Barts Health NHS, London, U.K.; \\ ${ }^{11}$ Experimental, Educational and Research Centre ELPEN, Athens, Greece; \\ ${ }^{12}$ School of Medicine, European University of Cyprus, Nicosia, Cyprus
}

\begin{abstract}
Aim: Essential Skills in the Management of Surgical Cases (ESMSC Marathon Course ${ }^{T M}$ ) Integrated Generation 4 (iG4) is the first reported multifaceted undergraduate surgical course aiming to provide holistic surgical teaching. In this prospective observational study, we explored students'views on the iG4 curriculum, and identified how it can potentially address modern challenges in surgical training. Material and Methods: Medical students were invited to apply to the course online and were screened against pre-defined criteria. A multi-national structured
\end{abstract}

This article is freely accessible online.

*These Authors contributed equally to this study.

Correspondence to: Michail Sideris, MD, MDRes, Ph.D., Women's Health Research Unit, Centre for Primary Care and Public Health, Blizard Institute, Barts and The London School of Medicine and Dentistry, Queen Mary University of London, Yvonne Carter Building, 58 Turner Street, London E1 2AB, United Kingdom. Tel: +44 2078822525, e-mail: m.sideris@qmul.ac.uk.

Key Words: Medical students, undergraduate medical education, curriculum, holistic surgical education, simulation training, professionalism. questionnaire incorporating five domains related to the course curriculum and our dedicated research network, was designed and distributed to participants after successful completion of the course. Results: Forty-one students from European and Asian medical schools completed the course and filled in the survey. The median overall evaluation score of the course was 4.73 out of 5 (interquartile range $=4.21$ 4.72) and all students found that $i G 4$ served the vision of holistic surgical education. ESMSC had a positive motivational effect towards following a career in surgery $(p=0.012)$ and $92.7 \%$ of students declared that it should be an essential part of a future medical school curriculum. There was no statistically significant difference $(p>0.05)$ in results between participants of different countries of study, year of studies or age group. Conclusion: The ESMSC Marathon Course $^{T M}$ is perceived as a unique course model, with an established educational value and a positive motivational effect towards surgery. It might potentially be implemented in future medical school curricula as an essential element of undergraduate surgical education. The iG4 curriculum has opened a new exciting horizon of opportunities for advancing undergraduate holistic surgical education.

Surgical training is evidently stepping into one of the most challenging chapters of its history, complicated by an ongoing 
economic recession and increasing pressures on current healthcare systems $(1,2)$. Such factors come hand in hand with staffing shortages and, subsequently, a lack of training opportunities (1). Additionally, increasing expectations for surgical novices, litigation and trending sub-specialisations in healthcare provision, in conjunction with emerging surgical technologies, predispose surgical education to a new set of challenges $(3,4)$. These changes have arguably led to an increase in workload and 'burnout' for trainees $(5,6)$, putting off talented aspiring surgeons from applying for specialty training, fostering unsatisfactory working environments and cultivating an increased dropout rate (7). Alas, tackling this situation has proven to be a true challenge, and initiatives to date have predominantly failed (2).

Several root cause analysis studies have attempted to identify the underlying problems and implement effective solutions, with the vast majority concluding that simulationbased learning (SBL) should be introduced as early as possible into the training pathway (8-10). Although SBL has a relatively high cost, often deemed to be unjustified, there is consensus that surgical skills training at the undergraduate level is beneficial, particularly in inspiring the future surgeon and allowing the development of solid technical proficiencies $(3,10)$. Furthermore, there is an emerging trend of nontechnical skill teaching, tailored to producing medical professionals of heightened patient responsibility and who can effectively perform in $21^{\text {st }}$ century organisational healthcare systems (11). This has become evident both at postgraduate as well as undergraduate level $(11,12)$. Nevertheless, numerous surgical courses are providing merely fragmented training, aimed either at technical or nontechnical skills. Moreover, hardly any of the modern programs emphasise dedicated basic science or applied surgical science modules, which leads to lack of critical thinking, particularly when a clinical scenario deviates from traditional guideline-based approaches.

Essential Skills in the Management of Surgical Cases (ESMSC-Marathon Course ${ }^{\mathrm{TM}}$ ) Integrated Generation 4 (iG4) is the first reported multifaceted surgical course, tailored for undergraduate students, aiming to provide holistic surgical teaching. We define 'holistic' as a curriculum which incorporates dedicated elements of basic and applied surgical science, combined with mixed fidelity technical and nontechnical skills, distributed across several surgical specialties in a clustered architecture. In this study, we primarily aimed to explore students' views on the iG4 curriculum as a novel tool for delivering holistic surgical education. Furthermore, we aimed to identify how a holistic educational model like iG4 can potentially address modern challenges in surgical training and to evaluate the role of our dedicated research network, as a novel means for engaging aspiring surgeons and continuously advancing the iG4 concept to meet the vision of 'holistic surgical education'.
ESMSC Medical Education Research Group (eMERG). This study was conducted as part of the eMERG, a multidisciplinary research group which is primarily dedicated to supply ESMSC with novel knowledge and advance its current curriculum (13). eMERG consists of a network of senior academics and consultants across Europe, along with junior and senior trainees who are expected to teach and inspire medical students in surgical education research.

Curriculum evolution. The ESMSC Marathon Course ${ }^{\mathrm{TM}}$ (esmsc.gr) was established in 2013. Curriculum evolution was based on a dynamic multifaceted model which incorporated input from students and faculty feedback $(\mathrm{F})(8)$, objective performance outcomes (OO) (12, 14-16), validated questionnaires on educational environment (V) (9), expert opinions (E) (13) and literature reviews (L) (10, 11). This approach has been previously described as the FOOVEL model (1). The latest version of the curriculum is referred to as Integrated Generation 4 (iG4) (1). The $\mathrm{iG} 4$ is the first reported three-dimensional (3D) curriculum architecture globally; its foundation principles reflect initially the transition from a mono-dimensional to a two-dimensional (2D) model, and finally to the latest $3 \mathrm{D}$ version, outlined below.

By the term mono-dimensional, we refer to a simple division of curriculum modules into either technical skills and knowledge workshops, or didactic lectures (single axis, version 14s) (1). Thereafter, a 2D version was introduced, which included a new axis, dividing the existing modules into four learning cores: Skills-based training, basic science, casebased learning, and applied surgical science (3). This model was represented as v20-15 and v20-15s (1), and was later upgraded to a newer version, Cores Integrated for Research (Ci4R). Despite this also being a 2D model, it contributed significantly towards establishing and refining the set-up of the course into four new learning cores: Technical skills, nontechnical skills, applied science, and basic science (1). Additionally, Ci4R was the first version which employed a structured research methodology, a process facilitated by the introduction of a novel dedicated research network, eMERG (13). The introduction of a third axis (specialty axis) was the foundation of the next and newest 3D curriculum version, the iG4. iG4 proof of concept has been described in detail (17). Even though it uses the same principles as the Ci4R, its modules are divided into 'specialty clusters'. Along the third axis, we also introduced objective parameters for each module i.e. heterogeneity in learning outcomes and specialty affinity allowing a mathematic description of the curriculum design, and subsequently the introduction of 'pragmatic outcomes'.

\section{Materials and Methods}

Study design. This was a prospective observational study. Participants were invited to apply for the course online by filling in a short survey 
form and providing their curriculum vitae (CV) and a personal statement. Forty-one participants were eventually enrolled in the study after fulfilment of pre-defined eligibility criteria: Good knowledge of the English language, and motivation and insight into surgery, after screening of their personal statement and CV. All participants completed the ESMSC Marathon Course ${ }^{\mathrm{TM}}$. During the course, participants attended a dedicated lecture on the iG4 novel curriculum and its primary aim and vision. Therefore, they were deemed familiar with the concept prior to completion of the post course questionnaire.

A structured online questionnaire was designed to meet the aims of this study (iG4 beta students' survey) and was distributed on completion of the last day of the course. The scope of the questionnaire was to identify whether our course curriculum fits with the vision of holistic education, and which elements are perceived as fundamental pillars for this purpose. We also included questions on how a holistic educational model like iG4 can potentially address modern challenges in surgical training. We finally included a cluster on the role of our dedicated research network (eMERG).

For this purpose, we developed a 5-domain survey tailored to address the aforementioned purpose. The survey consisted of five domains:

1: Demographics, focused to reflect the educational background of each student.

2: Role of the ESMSC course as a teaching and motivation-towardssurgery model.

3: Role of the iG4 model as a tool for holistic surgical education.

4: Evaluation of the learning component (cores) of the course.

5: Evaluation of our research network towards involving students in surgical education research.

Data were prospectively collected within $72 \mathrm{~h}$ using a single automated reminder at $48 \mathrm{~h}$.

Place of study. The ESMSC Marathon Course ${ }^{\mathrm{TM}}$ was developed at the Experimental Educational and Research Centre ELPEN (Athens, Greece). The first pilot application of the iG4 (beta version, series 11) ran at the Case for Advanced Simulation and Education (CASE), Acibadem University, Istanbul, Turkey. CASE provides advanced facilities in dry and wet laboratory SBL, and virtual hospital technologies. The current study is based on the $11^{\text {th }}$ series of the ESMSC at CASE.

Ethical approval. The ESMSC course is compatible with the current 3R principles for animal-model simulation (refinement, replacement and reduction). Application of ethical approval met directive 63/2010, PD 56/April 2013 declaration, according to local policy (license reference number is 4857/15-09-2017, MS, AP et al.) All procedures performed in studies involving human participants were in accordance with the ethical standards of the institutional and/or national research committee and with the 1964 Helsinki declaration and its later amendments or comparable ethical standards. All applicable international, national, and/or institutional guidelines for the care and use of animals were followed.

Data collection and statistical analysis. Data were collected online using Google Forms and were subsequently exported to a digital spreadsheet. Inferential univariate non-parametric statistics were used to describe our data; analysis was performed on IBM SPSS for Macintosh, Version 25.0 (IBM Corp., Armonk, NY, USA). Normality of data distributions was assessed with Shapiro-Wilks test. Internal consistency reliability of the survey data was assessed using Cronbach's Alpha coefficient and the Corrected Item-total Correlations (ICC). Internal consistency was considered acceptable when Cronbach's Alpha was between 0.5 and 0.7 , and good if above 0.7. The ICC was used to evaluate the level of agreement between measurements. ICC $<0.2$ is considered as poor agreement, 0.21-0.40 as fair, 0.61-0.80 as good and $0.81-1.0$ as very good (18).

\section{Results}

Demographics. Forty-one students attended the course: 27 (65.9\%) from United Kingdom medical schools (King's College London and Imperial College London), six (14.6\%) from the University of Athens, five (12.5\%) from Acibadem University (Istanbul, Turkey) and three (7.3\%) from Indian Medical Schools. Completion rate of the survey was $100 \%$. Five students $(12.2 \%)$ had completed a previous degree prior to medicine. Thirty-four $(82.9 \%)$ were aged $20-24$ years and seven $(17.1 \%)$ to $24-30$ years. Thirty-seven students $(90.2 \%)$ were in their clinical year rotations and four $(9.8 \%)$ in the preclinical years. There was no statistically significant difference ( $p>0.05)$ between scores for this domain from participants across different countries of study, year of studies or age group.

Motivation towards surgery. Thirty-five students $(85.4 \%)$ were interested in pursuing a career in surgery prior to attending the course, whereas on completion of the course all 41 delegates declared interest to pursue surgical training $(p=0.012)$. All students stated that ESMSC familiarised them with a holistic view of surgery and 40 of them $(97.6 \%)$ declared that it positively motivated them towards surgery. Thirty-eight students $(92.7 \%)$ felt that the ESMSC should be an essential part of a medical school curriculum and 40 (97.6\%) perceived ESMSC as a unique course concept. There was no statistically significant difference $(p>0.05)$ between scores for this domain from participants across different countries of study, year of studies or age group.

$i G 4$ as a tool for holistic surgical education. Twenty-three $(56.1 \%)$ of the students stated that the iG4 curriculum fit the vision of holistic surgical education, and 18 (43.9\%) believed it was a relatively good setup for this vision and that it provided a good overview of surgical education. None of the students felt ESMSC fell short of the vision of holistic surgical education. Twenty $(48.8 \%)$ participants found that the unique combination of wet and dry laboratory simulation, with advanced virtual hospital SBL and dedicated workshops on basic and applied surgical science to be a key element supporting the ESMSC vision. Twenty-nine participants $(70.7 \%)$ stated that they would 're-attend' the course and 12 $(29.3 \%)$ that they would 'maybe re-attend'. There was no statistically significant difference $(p>0.05)$ between scores for this domain from participants across different countries of study, year of studies or age group. 
Table I. Participant feedback for the $11^{\text {th }}$ series of Essential Skills in the Management of (ESMSC) Marathon Course ${ }^{T M}$.

\begin{tabular}{lrr}
\hline Question item (Likert Scale 1-5) & $\begin{array}{c}\text { Median* } \\
\text { Percentile }\end{array}$ & $\begin{array}{c}50 \% \\
\text { Percentile }\end{array}$ \\
\hline How do you rate your overall experience with the ESMSC course? & 4.725 & 4.213 \\
How do you rate the Basic Science workshops (ABGs, management of fluids and sepsis)? & 4.725 \\
How do you rate the In Vivo Wet Lab modules (laparoscopy, dissections on swine model)? & 4.441 & 4.677 \\
How do you rate the Dry Lab simulation modules (laparoscopic boxes, arthroscopy, & 4.902 & 4.402 \\
clinical skills lab, microsurgical skills)? & 4.825 & 4.313 \\
How do you rate the Ex Vivo simulation modules (suturing, ORIF, tendon repair, neurosurgical modules)? & 4.718 & 4.825 \\
How do you rate the Applied Surgical Science workshops and case-based discussions? & 4.472 & 3.842 \\
How do you rate the facilities of Centre of Advanced Simulation and Education? & 4.950 & 4.450 \\
How approachable were the faculty (technicians)? & 4.756 & 4.256 \\
How approachable were the faculty (instructors)? & 4.825 & 4.950 \\
How do you rate the faculty selection? & 4.805 & 4.313 \\
\hline
\end{tabular}

ABGs: Arterial blood gases; CASE: Centre for Advanced Simulation and Education; ORIF: Open Reduction Internal Fixation. *All comparisons across different educational backgrounds, age groups or stage of training resulted in $p>0.05$.

Participant feedback for each domain. Table I summarises the dedicated feedback across each domain. Comparing performance across different medical schools, stage of studies and age groups, there were no statistically significant difference in feedback ( $p>0.05$ for all associations). The median overall perception of the course was 4.73 out of 5 (interquartile range $=4.21-4.72$ ).

Involvement in surgical education research. Thirty-three $(80.5 \%)$ students declared they would 'be interested' in surgical education research, and seven $(17.1 \%)$ that 'may be interested'; only one (2.4\%) was 'not interested'. Similarly, $33(80.5 \%)$ of the students would apply to be considered for the eMERG research training network. There was no statistically significant difference $(p>0.05)$ between scores for this domain from participants across different countries of study, year of studies or age group.

Internal consistency reliability analysis. The Cronbach's Alpha coefficient based on standardised items was 0.855 (Cronbach's Alpha coefficient $=0.833$ ) indicating good internal consistency of the survey data. The mean ICC was 0.370 , in keeping with fair agreement between measurements.

\section{Discussion}

Key findings. There was no difference in the provided feedback across participants of different countries of study, including UK, Greek, Turkish and Indian medical schools. Similarly, no difference was noted between students of different age groups or year of study. Hence, students' views on the course seem to be unified and homogenous despite dissimilarities in their demographic and academic backgrounds. Such a conclusion confirms our initial assumption that the course is adaptable to a number of educational settings globally. The vast majority of students considered the ESMSC Marathon Course ${ }^{\mathrm{TM}}$ to be a unique concept with a positive motivational effect towards pursuing a career in surgery. Moreover, all learning cores of the course were highly rated and more than nine out of 10 students found the implementation of the ESMSC course in medical school curriculum to be appropriate. This not only demonstrates the value of the course as an educational tool, it also signals that in future implementation, it would indeed be positively accepted by students.

Notedly, students appear to be inspired by the novel concept of 'holistic surgical education' and acknowledged the ESMSC iG4 curriculum to be a stepping-stone in this direction. More than three-quarters of the students appreciated that identifying the most salient combination of surgical modules across the different cores, alongside a melange of mixed fidelity simulation modalities, is a key element for achieving a holistic curriculum. This statement enhances our initial speculations that the ESMSC Marathon Course $^{\mathrm{TM}}$ has a multifaceted character and multi-speciality components are arguably the key to achieving the vision of a holistic surgical curriculum.

More than eight out of 10 students stated they would be interested in joining eMERG and pursuing research involvement in surgical education. Although the ESMSC student cohort could be considered as already being exceptionally motivated, given that motivation was an application screening factor, this trend appears to be very promising. Engagement of more students in the research engine of the course (eMERG) could improve our insight into how students see our efforts from their perspective.

Interpretation - Are these results something new? The ESMSC is an already validated educational model which has 
gained excellent feedback and recognition $(1,3,8)$. We have also discussed the role of ESMSC in motivating students towards a surgical career (8), and many students have expressed interest in being part of the eMERG research network (13). We have also already assessed how the course is perceived across different educational backgrounds (9).

However, this is the first exploration of how the ESMSC model is unique, and which curriculum elements are the students' favourites. We concluded that the combination of a large number of different modules (technical and nontechnical skills, basic and applied surgical science workshops) alongside variations in the delivery methods (i.e. fidelity level, wet or dry laboratory) are perceived as the key elements of success behind the course. This survey has also demonstrated that the above process is what the students perceive as 'holistic surgical education', and, fortunately, what we as faculty perceive as the primary vision for the course. Furthermore, our results determined that modules involving a greater array of skills (i.e. virtual hospital or wet laboratory in vivo SBL) are gaining popularity as the highlights of the ESMSC setup. Such findings are further supported by previous studies concluding that in vivo simulation at the undergraduate level is indeed a valuable tool for inspiring and supporting aspiring surgeons $(15,16)$.

How can the iG4 curriculum change the landscape in surgical education? As discussed, modern surgical education is facing an oxymoron situation, where global economic recession burdens healthcare provision and affects educational opportunities, whilst at the same time there are increasing expectations for excellence in surgical care. This can be partially attributed to emerging advanced technologies, which are becoming an indispensable part of clinical care, and are underpinning more demanding continuing medical education and a steeper learning curve.

In this stressful landscape, there are certain parameters that can be adjusted to potentially tackle part of the problem. These are primarily targeted at the undergraduate and earlypostgraduate level by the introduction of a robust and standardised education system, which not only delivers the correct foundations, but also instils inspiration and promotes excellence in surgical care. Another remedy for this situation is acknowledging that all of our efforts as clinicians and researchers are focused on a single denominator: To improve patient care. Such a practice can be promoted by introducing more 'holistic' teaching elements into medical school curricula, which deliver technical and non-technical skills, and knowledge-based teaching in unison. Their combination is a prerequisite for practicing evidence-based medicine. To put all of the above in context, the iG4 curriculum used in the ESMSC Marathon Course ${ }^{\mathrm{TM}}$ can indeed lay new ground in surgical education. All of the driving forces behind its inception are focused on designing modules which encompass a well-rounded mixture of skills, which at the same time are able to function effortlessly as part of a welldesigned and organised curriculum framework, with a clear direction and room for continuous improvement.

Strengths and limitations of the study. Enrolling students from medical schools of different continents (Europe and Asia), and with a huge variation in their respective medical curricula, sets a representative sample. Furthermore, the prospective nature of the study allowed a robust sample to be collected without losing any data to follow-up. Finally, although not validated, the internal consistency reliability analysis indicated that our results have good reliability and fair internal agreement between different measurements, which subsequently demonstrated a well-designed survey.

We recognise a series of limitations: iG4 proof of concept has only undergone a single pilot implementation; future courses will allow us to draw further conclusions. Moreover, the study had a relatively limited sample size and all data were collected at a single institution. Finally, the survey was not validated, however, reliability analysis revealed a fair agreement between our measurements, as mentioned above.

Future directions. The future of the ESMSC course is built on three pillars: Adaptation and reproducibility in an international setting, fragmentation into discrete clusters, and the introduction of pragmatic educational outcomes. The vision is to achieve a blueprint for a global course which fully serves the idea of 'holistic surgical education'. One of the primary goals of iG4 is to become an internationally reproducible curriculum, applicable to any setting; one aspect in this direction is the idea of forming discrete specialty clusters, which logistics-wise would allow more cost-efficient and tailored running of the course depending on local needs. For instance, should a provider decide to run a certain part of the ESMSC curriculum, cluster fragmentation would effectively allow that without compromising the quality of the educational outcome.

A unique advantage of the iG4 perception is the potential of describing each module via a unique 3-parameter mathematic point $(\mathrm{x}, \mathrm{y}, \mathrm{z})$. This means that each curriculum cluster can be represented by a unique surface in 3D space; equally the whole curriculum can be represented by a mathematically defined volume in the $\mathrm{x}, \mathrm{y}, \mathrm{z}$ approach. The mathematic description of both cluster 'surface' and curriculum 'volume' can represent objectively pragmatic (or mathematically defined) outcomes. Needless to say, the same applies for each individual module, as a unique point alongside a certain combination of heterogeneity for $\mathrm{x}$ and $y$, and affinity of $z$, which can also define another set of pragmatic (or mathematically defined) outcomes.

Currently any description of course curriculum performance is based on descriptive outcomes, i.e. the 
provision of validated or unvalidated feedback scales, tailored to the needs of the setting. ESMSC would be the first reported course internationally to connect pragmatic outcomes, derived from iG4's 3D setup, and compare them with existing descriptive outcomes, revolutionising the current concept of curriculum design (19). This can be further enhanced by the introduction and rapid advancement of artificial intelligence in surgical education.

\section{Conclusion}

The ESMSC Marathon Course ${ }^{\mathrm{TM}}$ is perceived as a unique course model, with an established educational value and a positive motivational effect towards surgery. ESMSC might potentially be implemented in future medical school curricula as an essential element of undergraduate surgical education. Participants in the course were positive and receptive to the idea of holistic surgical education and found that iG4 can be the stepping-stone transition in this direction. Students appeared motivated to join our research initiative (eMERG) and showed interest in assuming an active role in a rapidly evolving educational landscape. In conclusion, the iG4 curriculum has opened up a new exciting horizon of opportunities for advancing undergraduate holistic surgical education.

\section{Conflicts of Interest}

The Authors declare that they have no conflicts of interest.

\section{Authors' Contributions}

Michail Sideris primarily conceived the idea of the Integrated Generation 4 ESMSC curriculum. Michail Sideris and Apostolos Papalois are the primary leads of the ESMSC Marathon Course ${ }^{\mathrm{TM}}$ and share ownership of the course concept. Iakovos Theodoulou and Marios Nicolaides contributed equally with Michail Sideris and drafted parts of the article. Michail Sideris, Iakovos Theodoulou, Marios Nicolaidesco-designed and approved research methodology (survey). Michail Sideris performed statistical analysis of the data. Elif Iliria Emin, Aikaterini Dedeilia, Efthymia Theodorou and John Hanrahancontributed in performing study and collecting the data. Ziena Abdullah and Funlayo Odejinmi provided feedback on the pilot iG4 interpretation. Professor Apostolos Papalois was the senior Author of this study, providing constructive feedback on the article. All Authors reviewed and approved the final article.

\section{Acknowledgements}

There were no funders to report for this submission. The Authors declare that they have no financial interest.

\section{References}

1 Sideris M, Hanrahan J, Tsoulfas G, Theodoulou I, Dhaif F, Papalois V, Papagrigoriadis S, Velmahos G, Turner P and
Papalois A: Developing a novel international undergraduate surgical masterclass during a financial crisis: Our 4-year experience. Postgrad Med J 94(1111): 263-269, 2018. PMID: 29519810. DOI: $10.1136 /$ postgradmedj-2017-135479

2 Orri M, Farges O, Clavien PA, Barkun J and Revah-Levy A: Being a surgeon-the myth and the reality: A meta-synthesis of surgeons' perspectives about factors affecting their practice and well-being. Ann Surg 260(5): 721-728; discussion 728-729, 2014. PMID: 25379843. DOI: 10.1097/SLA.0000000000000962

3 Sideris M, Papalois A, Tsoulfas G, Majumder S, Toutouzas K, Koletsis E, Dedeilias P, Lymperopoulos N, Papagrigoriadis S, Papalois V and Zografos G: Developing an international combined applied surgical science and wet lab simulation course as an undergraduate teaching model. Biomed Res Int 2015:463987, 2015. PMID: 26613083. DOI: 10.1155/2015/463987

4 Geiger JD and Hirschl RB: Innovation in surgical technology and techniques: Challenges and ethical issues. Semin Pediatr Surg 24(3): 115-121, 2015. DOI: 10.1053/j.sempedsurg.2015.02.008

5 Elmore LC, Jeffe DB, Jin L, Awad MM and Turnbull IR: National survey of burnout among US general surgery residents. J Am Coll Surg 223(3): 440-451, 2016. PMID: 27238875. DOI: 10.1016/j.jamcollsurg.2016.05.014

6 Antiel RM, Reed DA, Van Arendonk KJ, Wightman SC, Hall DE, Porterfield JR, Horvath KD, Terhune KP, Tarpley JL and Farley DR: Effects of duty hour restrictions on core competencies, education, quality of life, and burnout among general surgery interns. JAMA Surg 148(5): 448-455, 2013. PMID: 23325404. DOI: 10.1001/jamasurg .2013.1368

7 Forel D, Vandepeer M, Duncan J, Tivey DR and Tobin SA: Leaving surgical training: Some of the reasons are in surgery. ANZ J Surg 88(5): 402-407, 2018. DOI: 10.1111/ans.14393

8 Sideris M, Papalois A, Theodoraki K, Dimitropoulos I, Johnson EO, Georgopoulou EM, Staikoglou N, Paparoidamis G, Pantelidis P, Tsagkaraki I, Karamaroudis S, Potoupnis ME, Tsiridis E, Dedeilias P, Papagrigoriadis S, Papalois V, Zografos G, Triantafyllou A and Tsoulfas G: Promoting undergraduate surgical education: Current evidence and students' views on ESMSC international wet lab course. J Invest Surg 30(2): 71-77, 2017. PMID: 27611894. DOI: 10.1080/08941939.2016.1220652

9 Sideris MC, Papalois AE, Athanasiou T, Dimitropoulos I, Theodoraki K, Dos Santos FS, Paparoidamis G, Staikoglou N, Pissas D, Whitfield PC, Rampotas A, Papagrigoriadis S, Papalois V, Zografos G and Tsoulfas G: Evaluating the educational environment of an international animal model-based wet lab course for undergraduate students. Ann Med Surg 12:8-17, 2016. PMID: 27830064. DOI: 10.1016/j.amsu.2016.10.004

10 Theodoulou I, Nicolaides M, Athanasiou T, Papalois A and Sideris M: Simulation-based learning strategies to teach undergraduate students basic surgical skills: A systematic review. J Surg Educ 75(5): 1374-1388, 2018. PMID: 29422405. DOI: 10.1016/j.jsurg.2018.01.013

11 Nicolaides M, Cardillo L, Theodoulou I, Hanrahan J, Tsoulfas G, Athanasiou T, Papalois A and Sideris M: Developing a novel framework for non-technical skills learning strategies for undergraduates: A systematic review. Ann Med Surg 36: 29-40, 2018. PMID: 30370054. DOI: 10.1016/j.amsu.2018.10.005

12 Nicolaides M, Theodorou E, Hanrahan JG, Theodoulou I, Emin EI, Papalois A and Sideris M: Advancing medical students' nontechnical skills in a group-based setting. J Invest Surg: 1-5, 2019. PMID: 31035823. DOI: 10.1080/08941939.2019.1602691 
13 Sideris M, Hanrahan J, Staikoglou N, Pantelidis P, Pidgeon C, Psychalakis N, Andersen N, Pittaras T, Athanasiou T, Tsoulfas $\mathrm{G}$ and Papalois A: Optimizing engagement of undergraduate students in medical education research: The eMERG training network. Ann Med Surg 31: 6-10, 2018. PMID: 29922460. DOI: 10.1016/j.amsu.2018.05.008

14 Dhaif F, Paparoidamis G, Sideris M, Hanrahan J, Georgopoulou EM, Tsagkaraki I, Staikoglou N, Saeed F, Michail T, Tzavelas A, Kenanidis E, Potoupnis M, Tsiridis E and Papalois A: The role of anxiety in simulation-based dexterity and overall performance: Does it really matter? J Invest Surg 32(2): 164169, 2019. PMID: 29286827. DOI: 10.1080/08941939. 2017.1387624

15 Pantelidis P, Sideris M, Tsoulfas G, Georgopoulou EM, Tsagkaraki I, Staikoglou N, Stagias G, Psychalakis N, Tsitsopoulos P, Athanasiou T, Zografos G and Papalois A: Is invivo laparoscopic simulation learning a step forward in the undergraduate surgical education? Ann Med Surg 16: 52-56, 2017. PMID: 28413632. DOI: 10.1016/j.amsu.2017.01.025

16 Sideris M, Papalois A, Theodoraki K, Paparoidamis G, Staikoglou N, Tsagkaraki I, Koletsis E, Dedeilias P, Lymperopoulos N, Imprialos K, Papagrigoriadis S, Papalois V, Zografos $\mathrm{G}$ and Tsoulfas $\mathrm{G}$ : Introducing in vivo dissection modules for undergraduate level trainees: What is the actual benefit and how could we make it more efficient? Indian J Surg 80(1): 68-76, 2018. PMID: 29581688. DOI: 10.1007/s12262016-1563-1

17 Sideris M, Papalois V, Athanasiou T, Papagrigoriadis S, Pierides M, Velmahos G and Papalois A: A novel multifaceted course blueprint to support outcome-based holistic surgical education: The integrated generation 4 model (iG4). In Vivo 34(2): XXXXXX, 2020. DOI:

18 Bland JM and Altman DG: Cronbach's alpha. BMJ 314(7080): 572, 1997. PMID: 9055718. DOI: 10.1136/bmj.314.7080.572

19 Prideaux D: Abc of learning and teaching in medicine. Curriculum design. BMJ 326(7383): 268-270, 2003. PMID: 12560283. DOI: $10.1136 /$ bmj.326.7383.268
Received January 3, 2020

Revised January 20, 2020

Accepted January 21, 2020 